\title{
Microbiological Evaluation of Prepared/Cooked Foods in a HACCP Environment
}

\author{
Esteban Pérez, António Raposo, Rafael Millán, Esther Sanjuán, Conrado Carrascosa
}

Animal Pathology, Animal Production, Bromatology, Food Science and Technology Department, Veterinary Faculty, University of Las Palmas de Gran Canaria, Las Palmas de Gran Canaria, Spain.

Email: estebanpg@gmail.com

Received May $9^{\text {th }}, 2011$; revised June $27^{\text {th }}, 2011$; accepted July $4^{\text {th }}, 2011$.

\begin{abstract}
In the production of safe food, a multitude of factors and elements along the food chain are involved. For this study we have established three factors relative to the food that have the possibility of influence on its microbiology content: basic ingredients, conservation time in refrigeration and handling degree. In the samples we looked forward three types of germens: warning, witness and pathogen. We did not find significant differences in the groups considered with regard to the variable of reference.
\end{abstract}

Keywords: HACCP, Food Microbiology, Food Quality, Food Safety, Evaluation

\section{Introduction}

Microbiological control of the food production has as last purpose, give sure or innocuous, nourishing and tasty products. The microbiological innocuousness seems to be distant, still in the advanced countries. The food habits of our modern society have changed very much, owed especially to the economic and technological social development, giving like proved the appearance of new food and productive technologies, increasing the consumption of prepared and precooked foods [1], with the consistent increase of this type of establishments.

Once developed the product, microbiological analysis allows to verify somehow that the previous process is done correctly, setting the appropriate corrective measures in case of non-conformities that exist and meet the critical limits established by current legislation [2] and for the quality politics of the food operator.

The key for the microbiological control, both of the innocuousness and of the food alteration, resides in the knowledge and in the application of the microbial ecology principles. The environmental influence of type is looked abiotic (physics, chemical and technological) and the effects biotic (interactions between different agents of the same habitat).

Until the 80's, the failure of the means was evident restored to prevent the food infections as consequence of a strategy of erroneous prevention, that is to say a retro- spective interpretation. More ahead it has been significant the effort of the industries and the sanitary administrations, which left the approach of retrospective control based on the analysis of the final products, taking the strategy of the control ahead to guarantee the microbiological innocuousness of the food.

Microbiological testing is one of the potential tools that can be used to evaluate whether a food safety risk management system is providing the level of control it was designed to deliver and microbiological criteria are designed to determine adherence to GHPs (good handling practices) and HACCP (Hazard Analysis and Critical Control Points) (i.e., verification) when more effective and efficient means are not available [3].

Foods may be contaminated from the processing environment, and testing at this level can act as an early warning system with the risks being identified and controlled before pathogens enter the food itself [4].

The systematically universally accepted for the food control and innocuousness used in the food industries is the HACCP and as consequence, the hygienic legislation has suffered a deep modification. Both the Food Codex and the European Union gather regulation in the matter. In the application of HACCP, the use of microbiological testing is seldom an effective means of monitoring Critical Control Points [5]. Codex Alimentarius establish regulations respect microbiological criteria for foods [6] and Hygienic practice for precooked and cooked foods in 
mass catering [7]. In European Community it is Regulation $852 / 2004$ [8] that can be deeply in each country.

Regulation 2073/2005 [2] gives us concrete details for a correct auto control like that as maximum limits of warning microorganisms and witnesses. The microbiological criteria in this paper come from this regulation.

The microbiological analyses are basic for the systems of monitoring and to put in functioning the corrector measurements before the possible food dangers, performing vital importance the use of the scoreboards to verify the quality of the food, due to the fact that the technologies used for the detection of these scoreboards are easy and rapid, at the same time as sure and economic.

The continuous analysis of a sufficient number of samples throughout the time of production, allows to obtain a valid information about the dangers associated with the different precooked foods, though there must be chosen routines of work that harmonize efficiency of results with economy of means. In the present study there are gathered aspects of monitoring of the system HACCP consisting of the determination of marking microorganisms in precooked foods.

The technical specific and direct aims derivatives of the previous approach were the following ones:

1) compared study of the food risks of microbiological origin for handling level in the food preparation.

2) compared study of the level of food risk of microbiological origin for basic ingredients of the food.

3) compared study of the repercussion in the quality of the samples to different conservation periods and the handling degree in its preparation.

4) Influence of the time of conservation in refrigeration conditions $\left(6^{\circ} \mathrm{C}\right)$, in the analytical results.

$5)$ the microbiological condition (state) analyzes of prepared food in a HAPPC environmental.

The main goal of this study is to determine factors, grouped into different groups that affect a major or minor degree of microbiological contamination of food and verify the influence of a HACCP system with regard to this contamination.

\section{Material and Methods}

For this study were taken a whole of 68 samples of precooked meals, with complete culinary processes of collective restoration centres, which had a system of hygienic sanitary already well-established (HACCP). Two universitary lunchrooms, placed in different campus of the island of Gran Canaria in Spain were tested. The study was realized from 2008 until 2010 in establishments which serve 200 - 300 lunches of traditional food per day and the food conservation time was lower than 72 hours.

\subsection{Samples Collected}

The food was qualified in three groups attending to a common factor and we think that it was influential on the microbial ecology and its importance in the HACCP:

1) Basic ingredients (Table 1):

- Meats: with or without sauces. 13 samples were analyzed; $19.12 \%$ of the whole.

- Fish and seafood: with or without sauces. 8 samples were analyzed; $11.76 \%$ of the whole.

- Eggs: fresh or manufacturer with thermal treatment. 6 samples were analyzed; $8.82 \%$ of the whole.

- Ultrafrozen Precooked: of animal or vegetable origin. With thermal treatment like fritter or boiling. 6 samples were analyzed; $8.82 \%$ of the whole.

- Pastas and rice: with or without sauces. 7 samples were analyzed; $10.29 \%$ of the whole.

- Vegetables: with thermal complete treatment (boiling). 9 samples were analyzed; $13.25 \%$ of the whole.

- Salads and cold foods: without thermal treatment with or without sauces, principally based on raw vegetables. 11 samples were analyzed; $16.18 \%$ of the whole.

2) Conservation time in refrigeration $\left(6^{\circ} \mathrm{C}\right)$ (Table 2):

- Foods preserved even/untill 24 hours. 28 samples were analyzed; $41.18 \%$ of the whole.

- Foods preserved between 24 and 48 hours: 21 samples were analyzed; $30.88 \%$ of the whole.

- Foods preserved even 72 hours: 19 samples were analyzed; $27.94 \%$ of the whole.

3) Handling degree (Table 3):

- High handling (with sauces or without thermal treatment): multifaceted ingredients with or without thermal treatment. There are 25 samples; $36.76 \%$ of the whole.

- Intermediate handling (without sauces): multifaceted ingredients and foods based on broths. They are 29 samples; $42.65 \%$ of the whole.

- Scanty handling (ultrafrozen): Group of different composition based on animal or vegetables ingredients, with thermal treatment type fritter and/or boiling. 14 samples were analyzed; $20.59 \%$ of the whole.

\subsection{Microbiology}

Group A: eaten prepared without treatment thermal and eaten prepared with thermal treatment, which there take ingredients not submitted to thermal treatment.

Group B: eaten prepared with thermal treatment.

Group C: eaten prepared submitted to sterilization.

Group D: eaten prepared packed, based on raw vegetables.

We only used A, B and D groups.

Three types of germens were looked: warning, witness and pathogen. 
Table 1. Basic ingredients group.

\begin{tabular}{ccc}
\hline \multicolumn{3}{c}{ BASIC INGREDIENTS } \\
\hline GROUP & SAMPLES & \% SAMPLES \\
\hline Meats & 13 & 19.12 \\
Fish and seafood & 8 & 11.76 \\
Eggs & 6 & 8.82 \\
Ultrafrozen precooked & 6 & 8.82 \\
Pastas and rice & 7 & 10.29 \\
Vegetables & 9 & 13.25 \\
Salads and cold foods & 11 & 16.18 \\
\hline
\end{tabular}

Table 2. Conservation time in refrigeration group.

\begin{tabular}{ccc}
\hline \multicolumn{3}{c}{ CONSERVATION TIME IN REFRIGERATION $\left(\mathbf{6}^{\circ} \mathbf{C}\right)$} \\
\hline GROUP & SAMPLES & \% SAMPLES \\
\hline $\begin{array}{c}\text { Foods preserved even/until } \\
24 \text { hours }\end{array}$ & 28 & 41.18 \\
$\begin{array}{c}\text { Foods preserved between } \\
24 \text { and } 48 \text { hours }\end{array}$ & 21 & 30.88 \\
$\begin{array}{c}\text { Foods preserved even } \\
72 \text { hours }\end{array}$ & 19 & 27.94 \\
\hline
\end{tabular}

Table 3. Handling degree group.

\begin{tabular}{ccc}
\hline \multicolumn{3}{c}{ HANDLING DEGREE } \\
\hline GROUP & SAMPLES & \% SAMPLES \\
\hline $\begin{array}{c}\text { High handling (with sauces or } \\
\text { without thermal treatment) } \\
\text { Intermediate handling } \\
\text { (without sauces) } \\
\begin{array}{c}\text { Scanty handling } \\
\text { (ultrafrozen) }\end{array}\end{array}$ & 25 & 36.76 \\
\hline
\end{tabular}

The warning germens must help to judge the good functioning of the establishment and the procedure of self-control applied in the elaboration of prepared meals.

- Aerobic mesophiles: cultivated fields in standard Agar (APHA), (PCA Cultimed 413799.1210). Incubation 24 - 48 hours to $31^{\circ} \mathrm{C}$.

- Enterobacteriaceae Lactose +: cultivated fields in Red Neutral-violet Agar Bilis Crystal with Lactose (VRBL Cultimed 413746) Incubation 24 hours to $31^{\circ} \mathrm{C}$.

A content of germens witness of lack of hygiene up, will involve the review of the methods of alertness applied in the points of critical control.

- Escherichia coli: Cultivated fields in Broth Bile to the Green Brilliant 2\% (VB, Difco 0007-17-4). Pipes provided with bell Durham, incubated to $37^{\circ} \mathrm{C}$ during
24 hours.

- Stahylococcus aureus: It was sowed in Agar de Baird-Parker (BP) (Adsa-mike 1-030) by emulsion of yolk of egg with potassium tellurite (Adsa-mike 6-026), incubated in plates during $24-48$ hours, to $37^{\circ} \mathrm{C}$

Of excelling itself the limits established for the pathogenic germens the affected products will be withdrawn from the market and excluded from the human consumption.

Eaten prepared will not contain any other pathogenic microorganisms nor its toxins, in a quantity that concerns the consumers' health.

- Salmonella spp.: From the dilution 1/10 of peptone water we sowed $50 \mathrm{ml}$ of food. with Broth of Selenite and Cystine (Cs, Cultimed 413809), double concentration, after 24 hours from incubation to $37^{\circ} \mathrm{C}$, a few droplets were sowed by handle in Agar Hektoen.

- Listeria monocytogenes: It was sowed Broth Palcam (Cultimed 465383.0922), incubated during 24 - 48 hours to $31^{\circ} \mathrm{C}$.

The analytical determinations were inventories in plate and of presence/absence and realized from serial dilutions decimal from $1 / 10$ up to $1 / 1000000$.

\subsection{Data Analysis}

\subsubsection{Description of the Variables}

1) Categorical variables for group of food:

- NSD: growth is not detected.

2) Categorical variables to determine the handling degree:

- high handling: 1

- intermediate handling: 2

- scanty handling: 3

3) Categorical variables according to the conservation time:

- equal or minor to 24 hours: 1

- between 24 and 48 hours: 2

- up to 72 hours: 3

4) Variables according to the main ingredient:

- Ultrafrozen precooked: 1

- Ultrafrozen vegetables: 2

- fished: 3

- meats: 4

- rice and pastas: 5

- pulse and vegetables: 6

- eggs: 7

- salads and cold foods: 8

\subsubsection{Statistical Treatment}

The chi-squared test was used to contrast if each of the warning variables of contamination was associated with the variables of handling degree, conservation time and 
type of food. A contrast was considered to be significant when the corresponding $\mathrm{p}$-value was lower than 0.05 .

\section{Results and Discussion}

The presence was not detected of: Salmonella spp., Listeria monocytogenes, and Staphylococcus aureus, in none of the analyzed samples. Fact that coincides with other studies of similar characteristics that now we present. To specify that the presence of Staphylococcus aureus, if it has been detected by major frequency in food of collective restoration, it presence for the most part owes to a bad conservation and/or incorrect food handling.

38 samples of the analyzed whole are not tolerated. Some of the microbiological analyzed parameters are in disagreement with the proposed maximums, this is $55.9 \%$ of the whole of the analysed samples.

As for the main ingredient, which had major number of not tolerated samples it was the group of salads with 9 samples ( $81.8 \%$ of its group), followed very closely by the meat group with 8 samples (61.5\% of its group), nevertheless it was the group of the vegetables the one that obtained the minor number of not tolerated samples, 2 in whole (22.2\% of its group). Nevertheless the results were not significant with one $p$ value of 0.247 either.

As for the conservation time in refrigeration, the values of not allowed samples were more similar between groups, since the group 1 (eaten preserved in refrigeration up to 24 hours) had 14 samples not tolerated $(50 \%$ of its group), in the group 2 (preserved in refrigeration between 24 and 48 hours it had 12 samples not tolerated (52.4\% of its group) and the group 3 (eaten preserved in refrigeration up to 72 hours) obtained 13 samples not tolerated ( $68.4 \%$ of its group). As for the statistical study value of 0.425 did not turn out to be significant with one $\mathrm{p}$ either.

Finally as for the handling degree, was observed that the eaten ones with high handling had major number not tolerated, a whole of 17 samples ( $68.0 \%$ of its group).

Followed to these, there were the eaten ones of intermediate handling in which was obtained a whole of 14 samples $(48.3 \%$ of its group); below these two groups stayed the group of scanty handling where only 7 samples were qualified of not tolerated (50\% of its group). Neither obtained statistical significance (meaning) $(p<$ 0,306 ).

\section{Conclusions}

Significant differences do not exist in the groups considered with regard to the variable of reference.

The application of a HACCP system does not have to make us think that the nourishing dangers in this type of establishments have disappeared. Only improving the regular control of food production in collective restoration establishments, we could be safe of the minimization of the risks.

\section{REFERENCES}

[1] S. Altekruse, D. Street, S. Fein and A. Levy, "Consumer Knowledge of Foodborne Microbial Hazards and Food Handling Practices," Journal of Food Protection, Vol. 59, 1996, pp. 287-294.

[2] J. Linden, "Microbiological Criteria for Foodstuffs," Food Standard Agency, London, 15 November 2005.

[3] M. van Schothorst, M. H. Zwietering, T. Ross, R. L. Buchanan and M. B. Cole, "Microbiological Specifications for Foods (ICMSF)," Food Control, Vol. 20, No. 11, 2009, pp. 967-979. doi:10.1016/j.foodcont.2008.11.005

[4] R. B. Tompkin, "Environmental Sampling-A Tool to Verify the Effectiveness of Preventative Hygiene Measures," Mitteilungen aus Lebensmitteluntersuchung und Hygiene, Vol. 95, No. 1, 2004, pp. 45-51.

[5] National Advisory Committee on Microbiological Criteria for Foods, "Hazard Analysis and Critical Control Point Principles and Application Guidelines," Journal of Food Protection, Vol. 61, 1998, pp. 762-775.

[6] Codex (Codex Alimentarius Comisión), Principles for the Establishment and Application of Microbiological Criteria for Foods, CAC/GL 21-1997, 1997.

[7] Codex (Codex Alimentarius Commision), Code of Hygienic Practice for Precooked and Cooked Foods in Mass Catering, CAC/RCP 39-1993, 1993.

[8] Commission Regulation, "The Hygiene of Foodstuffs," Fresh Quality Guild, 29 April 2004. 